\title{
Colocalization of QTLs for hull-cracked rice and grain size in elite rice varieties in Japan
}

\author{
${\text { Kenji Fujino*1), }{ }^{*} \text { (uji Hirayama }}^{2)}$, Mari Obara ${ }^{1)}$ and Tomohito Ikegaya ${ }^{1)}$ \\ 1) Hokkaido Agricultural Research Center, National Agricultural Research Organization, Sapporo, Hokkaido 062-8555, Japan \\ 2) Rice breeding group, Kamikawa Agricultural Experiment Station, Local Independent Administrative Agency Hokkaido Research \\ Organization, Pippu, Hokkaido 078-0397, Japan
}

The control of insects that consume cereal grains is important for the production and storage of grains. Hullcracked rice, which has splits in the hull, becomes more susceptible to insects both in the paddy field and during storage. The development of varieties with a low frequency of hull-cracked rice is the most economical and effective strategy to avoid insect damage and the environmental risks from agricultural chemical entering rice grains. In this study, we identified that QTLs for the frequency of hull-cracked rice and for grain width are located on the same chromosome using recombinant inbred lines derived from a cross between the elite rice varieties in Hokkaido, Japan, which are from the same pedigree and are genetically closely related. These QTLs were detected close to different molecular markers, which were separated by 1,101,675 bp, on chromosome 5 in the reference Nipponbare genome. In addition, low coefficient values of the phenotype were found between hull-cracked rice and grain size. These results suggested that the ratio of hull-cracked rice is independent of grain size. Using these QTLs, new varieties with low hull-cracked rice could be developed regardless of grain size.

Key Words: elite variety, grain size, hull-cracked rice, myINDEL, QTLs, rice.

\section{Introduction}

Rice grains are susceptible to insect damage. During the ripening stage in the paddy field, rice grains are injured by rice leaf bug Trigonotylus caeestialium, decreasing the grain quality at market in Japan (Ishimoto 2007, Ito 2004). Worldwide, stored rice is susceptible to the lesser grain borer Rhyzoperha dominica (Chanbang et al. 2008a, 2008b, Kavallieratos et al. 2012). Rice grains are enclosed in a hull formed by two leaves, palea and lemma. Hull-cracked rice, which has splits in the hull, becomes more susceptible to grain borer attack. Nymphal development depends on feeding on grains with cracked hulls. The appearance and frequency of hull-cracked rice may reflect in the density of T. caeestialium in the field (Ishimoto 2007, Ito 2004), while $R$. dominica selects grains with hull-cracked rice for reproduction (Kavallieratos et al. 2012).

Damage from the insects can cause significant reductions in yields. Agricultural chemical control of insects has been

Communicated by Hei Leung

Received March 6, 2018. Accepted June 5, 2018.

First Published Online in J-STAGE on August 8, 2018.

*Corresponding author (e-mail: kfujino@affrc.go.jp) used in rice cultivation, but being able to omit chemical use is important for food safety in the production of grain cereals, the cost of chemicals, and environmental concerns. Development of varieties with lower frequencies of hullcracked rice is the most economical and effective strategy to avoid insect damage and environmental risks from chemical use in rice. However, rice breeding programs for hullcracked rice are difficult because of inadequate knowledge of the genetic basis of this trait.

Imbalance between hull size (sink) and grain size (source) may generate hull-cracked rice. Increased grain size is major objectives in rice breeding programs, and spikelet hulls could restrict grain growth, thus determining grain size (Li and Li 2016). Many QTLs for grain size have been identified for diverse grain size traits (reviewed by Huang et al. 2013), such as the difference between subspecies levels, indica-japonica. Today, the number of QTLs for grain size is increasing (Zhao et al. 2017, Zhen et al. 2017). Therefore, it is unclear which genes play a major role in the control grain size in the local rice varieties.

Current elite rice varieties in Japan show high grain quality and good eating quality for the market in Japan. Based on the grain shape, rice is divided into three classes, long, medium, and short, for which (grain length)/(grain width) 
values are more than 3.0, 2.0-3.0, and smaller than 2.0, respectively. Preferences for grain shapes in rice depend on the cultures in local regions (Calingacion et al. 2014). Short, round grains are favored in Japan, Korea, and northern China. Rice breeding programs have established many varieties with high yield and good eating quality in Hokkaido, Japan (Fujino et al. 2017). Elite rice varieties tend to have many tillers with short panicles. Without a change in grain size, rice production, as measured using 1,000-grain weight, has improved during rice breeding programs.

QTL mapping is an effective way to dissect the genetic basis of quantitative traits, including most of agronomic traits. QTLs for various agronomic traits have been identified during the past two decades (http://www.gramene.org.). Many types of molecular marker sets, restriction fragment length polymorphisms (RFLPs), simple sequence repeats (SSRs), insertion/deletions (InDels), and single nucleotide polymorphisms (SNPs) have been listed. However, the efficiency of the application of these markers in studies, in which polymorphisms have been detected within materials, are dependent on the genetic relationships between varieties. The set-up of molecular markers that are polymorphic between parental lines is a key step for performing QTL mapping, but this is time consuming and labor-intensive when the parents are genetically closely related. In the pedigree of Japanese modern rice varieties, a small number of varieties and superior landraces have been frequently used as parental varieties (Yamamoto et al. 2010). This efficiency directly facilitates QTL analysis as genetic studies. Recently, several kinds of SNP chips have been used (Shanmugavadivel et al. 2017, Singh et al. 2017, Wang et al. 2017). In addition, genotype-by-sequencing (GBS) by next-generation sequencing (NGS) has been applied in rice (Chen et al. 2016, De Leon et al. 2016, Furuta et al. 2017, Zaid et al. 2017), because NGS has enabled the discovery of numerous SNPs. However, there is the cost of laboratory research on QTL analysis using primary mapping populations. Furthermore, there are too many datasets for breeders to handle alone.

Here, we reported QTLs for hull-cracked rice in rice. The frequency of hull-cracked rice has been increasing in elite rice varieties in Hokkaido, Japan, during rice breeding programs (Fujino et al. 2017). Using recombinant inbred lines (RILs) between the elite varieties in Hokkaido, a single QTL with a large effect on hull-cracked rice has been identified on chromosome 5. In addition, QTL analyses for grain size, length, width, and thickness were carried out. Another QTL on chromosome 5 increased grain width and thickness. Finally, we propose an analysis flow for NGS data for customizing DNA marker development for laboratory research, which was named myINDEL.

\section{Materials and Methods}

\section{Plant material}

Two japonica elite rice varieties, Hoshinoyume (HS) and
Kitakurin (KK), were used in this study as the parental varieties. Both were bred in rice breeding programs in Hokkaido, Japan, for the same current market class in Japan. HS was registered in 1996 and it shows a high frequency of hull-cracked rice. KK is a progeny of HS and was registered in 2014, but it has a low frequency in hull-cracked rice (Supplemental Fig. 1). To identify QTLs controlling hullcracked rice and grain size, we developed mapping populations of RILs derived from the cross between HS and KK.

\section{Phenotype evaluation}

Four agronomic traits were evaluated in accordance with Fujino et al. (2017). In brief, the frequency of hull-cracked rice, grain length, grain width, and grain thickness were evaluated. We evaluated the values of varieties and RILs over two years, 2013 and 2014, because agro-economical traits are influenced by environmental conditions such as temperature during growth periods.

Plant material (Supplemental Fig. 1), re-sequencing, DNA analysis (Supplemental Table 1), and QTL analysis are described in Supplemental Text 1.

\section{Results}

Variations in the four traits of the ratio of hull-cracked rice and grain size

There was a large difference in the frequency of hullcracked rice between the varieties (Fig. 1, Table 1). HS showed high levels of hull-cracked rice, $62.0 \%$ in 2013 and $71.5 \%$ in 2014, whereas KK showed low levels of hullcracked rice, $2.2 \%$ in 2013 and $4.8 \%$ in 2014. In contrast, the differences in grain size were small and the grain shapes

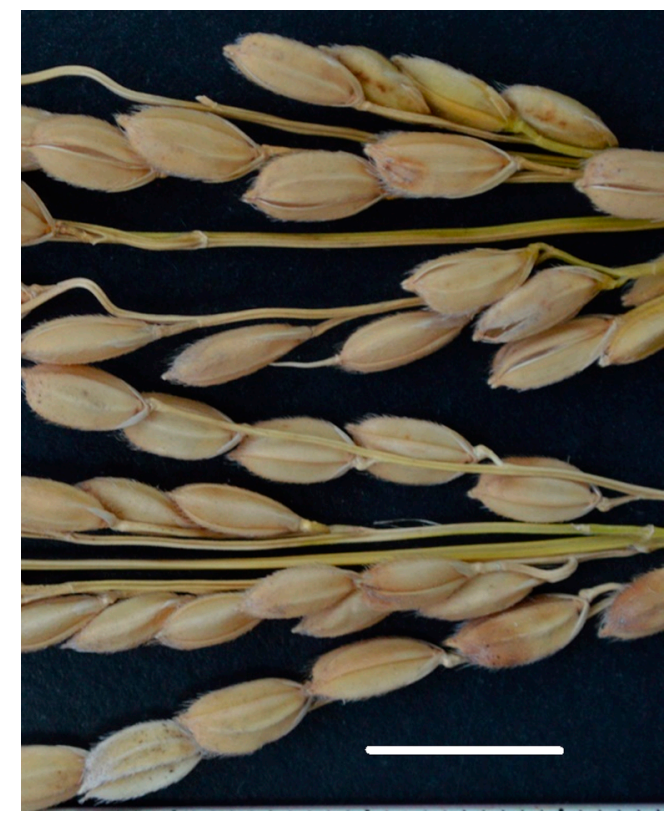

Fig. 1. Panicles with a high ratio of hull-cracked rice in Hoshinoyume (HS, upper) and with a low ratio in Kitakurin (KK, lower). Scale indicates $1 \mathrm{~cm}$. 
belonged to the same market class, 1.89 of HS and 1.77 of $\mathrm{KK}$ in (grain length)/(grain width) ratio (Table 1).

To identify QTLs controlling hull-cracked rice and grain size, we developed mapping populations of RILs derived from the cross between HS and KK. There was wide variation with a continuous distribution in the frequency of hullcracked rice, ranging from $2.2 \%$ to $71.5 \%$ (Fig. 2, Supplemental Table 2). Conversely the variations in grain size, grain length, grain width, and grain thickness were all small. High coefficients of phenotype between years, 0.75-0.81, were detected in all four traits, suggesting that these traits were stable for environmental conditions. There was a low coefficient between hull-cracked rice and grain size (Supplemental Table 3). Among the traits for grain size, low coefficients were observed between grain length and grain width and between grain length and grain thickness, whereas high positive coefficients were detected between grain width and grain thickness, 0.66 in 2013 and 0.76 in 2014 (Supplemental Table 3). Therefore, (grain length)/(grain width) of grain shape in the RILs showed that they were in the same market class, with narrow ranges of $1.68-1.98$ in 2013 and 1.70-2.00 in 2014.

Table 1. Parental phenotypes

\begin{tabular}{lccc}
\hline \hline Trait & Year & $\begin{array}{c}\text { Kitakurin } \\
(\mathrm{KK})\end{array}$ & $\begin{array}{c}\text { Hoshinoyume } \\
(\mathrm{HS})\end{array}$ \\
\hline Hull-cracked rice (\%) & 2013 & 2.2 & 62 \\
& 2014 & 4.8 & 71.5 \\
\hline Grain length (mm) & 2013 & $5.18 \pm 0.23$ & $5.28 \pm 0.25$ \\
& 2014 & $5.21 \pm 0.28$ & $5.25 \pm 0.28$ \\
\hline Grain width (mm) & 2013 & $2.93 \pm 0.19$ & $2.79 \pm 0.14$ \\
& 2014 & $2.97 \pm 0.17$ & $2.84 \pm 0.13$ \\
\hline Grain thickness (mm) & 2013 & $1.98 \pm 0.11$ & $1.94 \pm 0.14$ \\
& 2014 & $1.99 \pm 0.15$ & $1.98 \pm 0.08$ \\
\hline Grain length/Grain width & 2013 & 1.77 & 1.89 \\
& 2014 & 1.75 & 1.85 \\
\hline
\end{tabular}

A
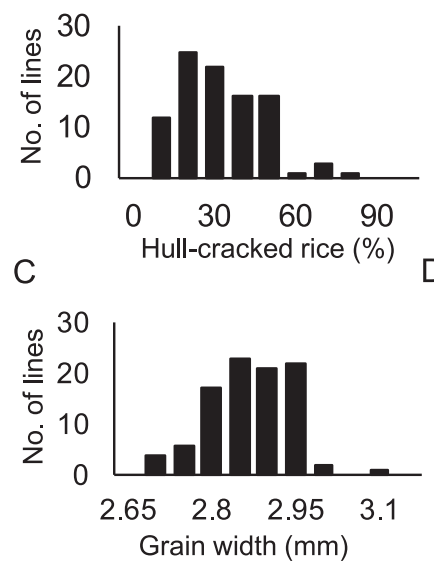

B
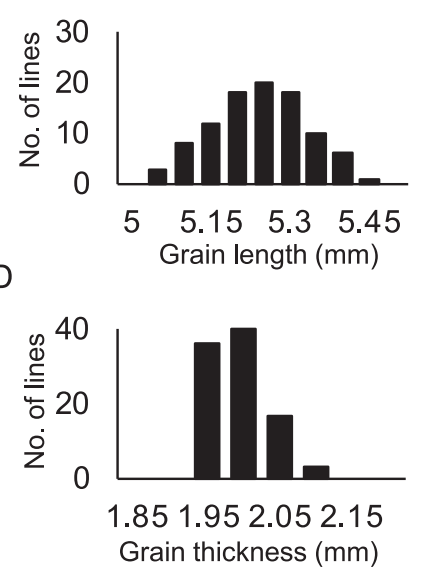

Fig. 2. Frequency distributions of hull-cracked rice and grain size in recombinant inbred lines $(n=96)$ derived from the cross between Hoshinoyume (HS) and Kitakurin (KK). A, the ratio of hull-cracked rice, B, grain length, C, grain width, D, grain thickness evaluated in 2013.

\section{Polymorphisms between the parental genomes}

To develop molecular markers for the parents, the genome sequences of HS and KK were determined using NGS methods. A total of $152.4 \mathrm{Mb}$ of reads in HS and $150.7 \mathrm{Mb}$ of reads in KK were obtained (Supplemental Table 4). Trimmed sequences were then mapped to the Nipponbare sequence (IRGSP 1.0). Finally, we had sequences, which covered more than $90 \%$ of the rice genome in $>10$ depth (Supplemental Table 4). SNPs were distributed over the genome (Supplemental Table 5). The distributions of SNPs varied along chromosomes, ranging from 7,750 on chromosome 5 to 38,807 on chromosome 11 in HS and from 8,457 on chromosome 5 to 37,214 on chromosome 11 in KK.

A total of 360,038 SNPs between HS and KK were distributed over the genome, ranging from 11,914 on chromosome 5 to 56,076 on chromosome 11 (Supplemental Table 6). Although the average SNP density per $1 \mathrm{Mb}$ was 965.0 over the genome, the distributions of SNPs varied along the chromosomes, ranging from 397.1 on chromosome 5 to 1933.7 on chromosome 11 (Fig. 3, Supplemental Table 7). The SNP density per $1 \mathrm{Mb}$ along the chromosomes was compared. The highest density of SNPs was 6,854 , located in the $16-17 \mathrm{Mb}$ region on chromosome 2,

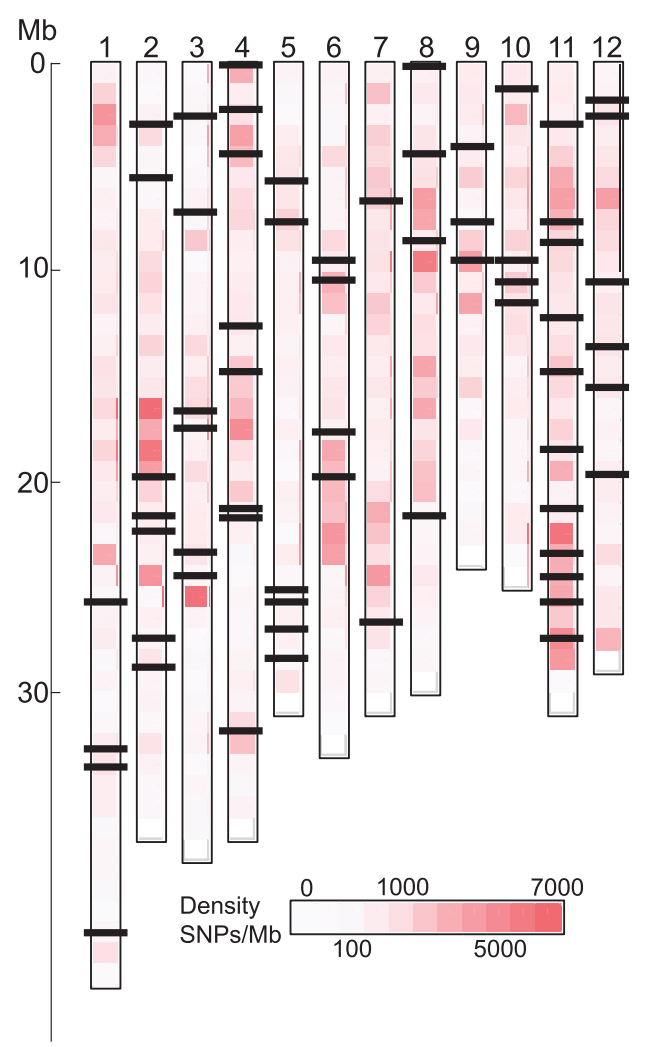

Fig. 3. SNPs distribution between Hoshinoyume (HS) and Kitakurin (KK) over the genome. Horizontal bars indicate the INDEL markers used for QTL analysis. Numbers (1-12) indicate rice chromosome numbers. The frame map is based on the Nipponbare genome (IRGSP 1.0). 
whereas the lowest density was 39 observed in the 40 $41 \mathrm{Mb}$ region on chromosome 1 (Supplemental Table 7). Although HS and KK, a progeny of HS, are genetically closely related, SNPs on the chromosomal regions confirmed their identity at the sequence level. The chromosomal regions with high and low SNP identity might be from different origins and shared origins, respectively. The longest chromosomal regions with SNPs more than the chromosomal averages were two $6-\mathrm{Mb}$ long sections in the $3-8 \mathrm{Mb}$ region on chromosome 5 and the $18-24 \mathrm{Mb}$ region on chromosome 6 , whereas the longest region with less than the chromosomal average was the $14 \mathrm{Mb}$ length in the 7-23 Mb region on chromosome 12 (Supplemental Table 7).

Next, polymorphic InDels, except for SSRs, between HS and KK over the genome were targeted for primer design for PCR as molecular markers. Among the 36,736 InDels in HS and 36,315 in KK (Supplemental Table 8), we focused on InDels of 20-2,000 bp in size over the genome, which are easier to handle in experiments in the laboratory. Sixtyfive InDel markers showed clear polymorphisms between the parents, and these markers were used for QTL analysis (Supplemental Table 1).

\section{QTLs with large effects on the frequency of hull-cracked rice and grain size}

A single QTL with a large effect on the frequency of hull-cracked rice was detected near INDEL01046 on chromosome 5, qHC5, which accounted for $20.0 \%$ in 2013 and $21.9 \%$ in 2014 of total phenotypic variation. The KK allele decreased the ratio of hull-cracked rice (Table 2).

A total of six QTLs for grain size were detected between the two years (Table 2). A QTL, $q G W 5$, with large effects, $23.5-31.6 \%$ of total phenotypic variation, for grain width and grain thickness was detected at INDEL01608 on chromosome 5 in both years. The KK alleles increased grain width and thickness. A single QTL, $q$ GL11, for grain length was detected at INDEL01036 on chromosome 11 in 2014 only. This QTL accounted for $21.4 \%$ of total phenotypic variation. The KK allele decreased grain length.

Both QTLs for hull-cracked rice and grain width, $q H C 5$ and $q G W 5$, were detected at the $26.0-28.0 \mathrm{Mb}$ region on chromosome 5 (Table 2, Supplemental Fig. 2).

\section{Discussion}

The control of insects in cereal grains is important for the production and storage of cereal grains. Hull-cracked rice, which has splits in the hull, becomes more susceptible to insects both in the rice field and during storage (Chanbang et al. 2008a, 2008b, Ishimoto 2007, Ito 2004, Kavallieratos et al. 2012). The development of varieties with low rates of hull-cracked rice is the most economical and effective strategy to avoid insect damage and reduce the environmental risks from chemicals applied to eliminate these insects on rice. In this study, we identified a QTL for hull-cracked rice near INDEL01046, $q H C 5$, and a QTL for grain width near INDEL01608, $q G W 5$, located on chromosome 5, and these QTLs were detected close to different molecular markers, which were separated by $1,101,675 \mathrm{bp}$ in the reference Nipponbare genome. There were 10 plants with recombinations between these markers (Supplemental Fig. 2, Supplemental Table 9). In addition, there were low coefficient values of the phenotype between hull-cracked rice and grain size (Supplemental Table 3). These results suggested that hull-cracked rice is independent of grain size, but these QTLs located in a $1.1 \mathrm{Mb}$ region on chromosome 5. The KK allele showed the opposite functions on the traits, a decrease in the frequency of hull-cracked rice at $q H C 5$ and enlargement of grain width at $q G W 5$.

QTLs for grain width and thickness, $q G W 5$ and $q G T 5$, were detected on the same chromosomal region near INDEL01608 on chromosome 5. There is a possibility that $q G W 5$ for grain width had pleiotropic effects on grain thickness. QTLs with pleiotropic effects on grain shape have been found frequently in rice (Bai et al. 2010, Fan et al. 2006, Li et al. 2011, Mao et al. 2010, Song et al. 2007, Zhen

Table 2. QTLs for hull-cracked rice and grain size

\begin{tabular}{|c|c|c|c|c|c|c|c|c|c|}
\hline Trait & Year & QTL & NMM & Chromosome & Position & LOD & PVE (\%) & $\mathrm{AE}$ & Threshold \\
\hline Hull-cracked rice $(\%)$ & $\begin{array}{l}2013 \\
2014 \\
\end{array}$ & $\begin{array}{l}\text { qHC5 } \\
\text { qHC5 }\end{array}$ & $\begin{array}{l}\text { INDEL01046 } \\
\text { INDEL01046 }\end{array}$ & $\begin{array}{l}5 \\
5 \\
\end{array}$ & $\begin{array}{l}26,862,898 \\
26,862,898 \\
\end{array}$ & $\begin{array}{l}7.35 \\
7.47 \\
\end{array}$ & $\begin{array}{l}20.0 \\
21.9 \\
\end{array}$ & $\begin{array}{r}-7.30 \\
-8.00 \\
\end{array}$ & $\begin{array}{l}4.54 \\
5.14 \\
\end{array}$ \\
\hline Grain length (mm) & $\begin{array}{l}2013 \\
2014\end{array}$ & $\begin{array}{l}q G L 11 \\
q G L 4\end{array}$ & $\begin{array}{c}- \\
\text { INDEL01086 } \\
\text { INDEL01036 }\end{array}$ & $\begin{array}{r}11 \\
4 \\
\end{array}$ & $\begin{array}{l}22,018,392 \\
13,370,937\end{array}$ & $\begin{array}{l}6.32 \\
3.27 \\
\end{array}$ & $\begin{array}{l}21.4 \\
10.0 \\
\end{array}$ & $\begin{array}{r}-0.050 \\
0.034 \\
\end{array}$ & $\begin{array}{l}3.45 \\
3.24\end{array}$ \\
\hline Grain width (mm) & 2013 & $\begin{array}{l}q G W 5 \\
q G W 2 \\
q G W 4 \\
q G W 11 \\
q G W 5 \\
q G W 2\end{array}$ & $\begin{array}{l}\text { INDEL01608 } \\
\text { INDEL01022 } \\
\text { INDEL01033 } \\
\text { INDEL01082 } \\
\text { INDEL01608 } \\
\text { INDEL01022 }\end{array}$ & $\begin{array}{r}5 \\
2 \\
4 \\
11 \\
5 \\
2\end{array}$ & $\begin{array}{r}27,964,573 \\
28,432,250 \\
695,303 \\
9,156,370 \\
27,964,573 \\
28,432,250\end{array}$ & $\begin{array}{r}8.29 \\
5.22 \\
3.87 \\
3.88 \\
12.13 \\
5.13\end{array}$ & $\begin{array}{r}23.5 \\
14.2 \\
2.3 \\
4.9 \\
31.6 \\
11.3\end{array}$ & $\begin{array}{r}0.034 \\
0.029 \\
-0.012 \\
-0.018 \\
0.040 \\
0.024\end{array}$ & 3.35 \\
\hline Grain thickness (mm) & $\begin{array}{l}2013 \\
2014\end{array}$ & $\begin{array}{l}q G T 5 \\
q G T 5 \\
q G T 2\end{array}$ & $\begin{array}{l}\text { INDEL01608 } \\
\text { INDEL01608 } \\
\text { INDEL01022 }\end{array}$ & $\begin{array}{l}5 \\
5 \\
2\end{array}$ & $\begin{array}{l}27,964,573 \\
27,964,573 \\
28,432,250\end{array}$ & $\begin{array}{l}6.95 \\
9.11 \\
4.49\end{array}$ & $\begin{array}{l}28.1 \\
26.2 \\
16.7\end{array}$ & $\begin{array}{l}0.021 \\
0.021 \\
0.018\end{array}$ & $\begin{array}{l}3.40 \\
3.42\end{array}$ \\
\hline
\end{tabular}

NMM; nearest molecular marker, PVE; phenotypic variation explained, AE; additive effect

Position is based on the Nipponbare genome (IRGSP 1.0). Threshold was culculated using 1,000 permutation tests. 
et al. 2017). Additionally, advanced backcrossed progenies derived from a single combination between japonica Koshihikari and indica IR64 confirmed the complexity of the control of grain size, with 65 QTLs identified (Nagata et al. 2015). Conventional breeding to develop improved varieties with low levels of hull-cracked rice has been conducted. The QTLs, $q H C 5$ and $q G W 5$, found in this study could have great potential value in rice breeding programs. Using these QTLs, new varieties with lower levels of hull-cracked rice could be developed instead of changes in grain size.

In this study, we performed QTL analysis using elite rice varieties in a local region that is genetically closely related. SSR markers among several kinds of DNA markers are useful for genetic studies in rice because they frequently show multiple alleles (McCouch et al. 2002). Considerable efforts in screening for polymorphic SSR markers between such varieties are critical for QTL analysis. To simplify such efforts, we can directly compare the genome sequences obtained from NGS. Without abundant SNPs, small numbers of polymorphic InDels could be sufficient for QTL analysis using primary mapping population. The significant impact of InDel markers is the easier manipulation by PCR methods in the laboratory.

We proposed this analysis flow as myINDEL, which customizes DNA marker development using NGS sequences in varieties that are genetically closely related (Fig. 4). At first, InDel regions over the genome were predicted by programs (for example Pindel (Ye et al. 2009) and Breakdancer (Chen et al. 2009)). Second, InDels were chosen based on the demands, the chromosomal position, polymorphism, and InDel

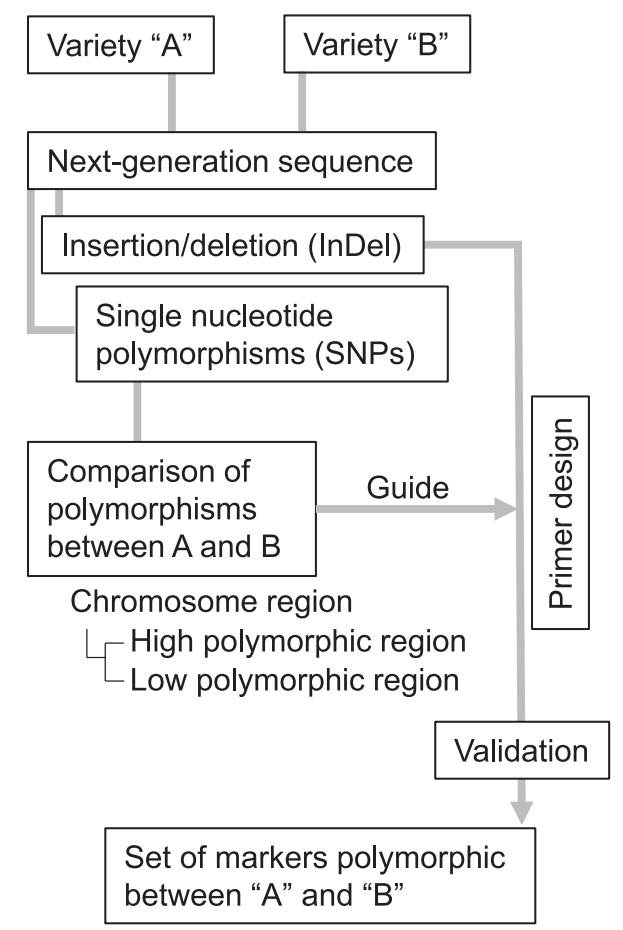

Fig. 4. myINDEL, an analysis flow for customizing the development of DNA markers between varieties that are genetically closely related. size. Third, primers were designed. Finally, polymorphism of InDel was validated by the experiment. myINDEL is breeder-friendly for the handling of huge data sets from NGS and could facilitate the identification of QTLs for sophisticated traits in elite varieties used in plant breeding programs. The dataset of SNPs in NGS sequences is too large to use for QTL analysis with the primary mapping populations, but is a way to detect polymorphism between varieties that are genetically closely related. The validated InDel markers will be used for not only QTL analysis but also in further studies including marker-assisted selection, fine mapping, and map-based cloning.

Elite traits with favorable values have been improved using germplasm within the similar genetic pool, or sometimes using exotic germplasm, in plant breeding programs (Fujino et al. 2015, 2017, Shinada et al. 2014). Associations between QTLs for elite traits and chromosomal genotypes may be from genetically different origins or by spontaneous mutations generated during rice breeding programs within identical chromosomal regions, such as loss-of-function $h d 5$ controlling heading date in rice (Fujino et al. 2013) and low temperature germinability $q L T G 3-1$ (Fujino and Iwata 2011, Fujino and Sekiguchi 2011). Improved understanding of the genetic basis of elite traits could improve the strategies to develop further elite varieties.

\section{Acknowledgments}

This work was supported in part by a grant from the Ministry of Agriculture, Forestry and Fisheries of Japan (Science and Technology Research Promotion Program for Agriculture, Forestry, Fisheries and Food Industry).

\section{Literature Cited}

Bai, X., L.Luo, W. Yan, M.R. Kovi, W.Zhan and Y.Xing (2010) Genetic dissection of rice grain shape using a recombinant inbred line population derived from two contrasting parents and fine mapping a pleiotropic quantitative trait locus $q$ GL7. BMC Genet. 11: 16.

Calingacion, M., A.Laborte, A. Nelson, A. Resurreccion, J.C. Concepcion, V.D.Daygon, R.Mumm, R.Reinke, S.Dipti, P.Z. Bassinello et al. (2014) Diversity of global rice markets and the science required for consumer-targeted rice breeding. PLoS ONE 9: e85106.

Chanbang, Y., F.H.Arthur, G.E. Wilde and J.E. Throne (2008a) Control of Rhyzopertha dominica in stored rough rice through a combination of diatomaceous earth and varietal resistance. Insect Sci. 15: 455-460.

Chanbang, Y., F.H.Arthur, G.E.Wilde and J.E. Throne (2008b) Hull characteristics as related to susceptibility of different varieties of rough rice to Rhyzopertha dominica (F.) (Coleoptera: Bostrichidae). J. Stored Prod. Res. 44: 205-212.

Chen,K., J.W.Wallis， M.D.McLellan， D.E.Larson， J.M.Kalicki, C.S.Pohl, S.D.McGrath, M.C.Wendl, Q.Zhang, D.P.Locke et al. (2009) BreakDancer: an algorithm for high-resolution mapping of genomic structural variation. Nat. Methods 6: 677-681.

Chen, L., W. Gao, S. Chen, L. Wang, J.Zou, Y.Liu, H. Wang, Z. Chen and T. Guo (2016) High-resolution QTL mapping for grain appearance 
traits and co-localization of chalkiness-associated differentially expressed candidate genes in rice. Rice 9: 48.

DeLeon, T.B., S.Linscombe and P.K. Subudhi (2016) Molecular dissection of seedling salinity tolerance in rice (Oryza sativa L.) using a high-density GBS-based SNP linkage map. Rice 9: 52.

Fan, C., Y.Xing, H.Mao, T.Lu, B.Han, C.Xu, X.Li and Q.Zhang (2006) GS3, a major QTL for grain length and weight and minor QTL for grain width and thickness in rice, encodes a putative transmembrane protein. Theor. Appl. Genet. 112: 1164-1171.

Fujino, K. and N. Iwata (2011) Selection for low-temperature germinability on the short arm of chromosome 3 in rice cultivars adapted to Hokkaido, Japan. Theor. Appl. Genet. 123: 1089-1097.

Fujino, K. and H.Sekiguchi (2011) Origins of functional nucleotide polymorphisms in a major quantitative trait locus, $q L T G 3-1$, controlling low-temperature germinability in rice. Plant Mol. Biol. 75: $1-10$.

Fujino, K., U. Yamanouchi and M. Yano (2013) Roles of the Hd5 gene controlling heading date for adaptation to the northern limits of rice cultivation. Theor. Appl. Genet. 126: 611-618.

Fujino, K., M. Obara, T. Ikegaya and K. Tamura (2015) Genetic shift in local rice populations during rice breeding programs in the northern limit of rice cultivation in the world. Theor. Appl. Genet. 128: 1739-1746.

Fujino, K., T.Nishimura, H.Kiuchi, Y.Hirayama and T. Sato (2017) Phenotypic changes during 100-year rice breeding programs in Hokkaido. Breed. Sci. 67: 528-534.

Furuta,T., M.Ashikari, K.K. Jena, K.Doi and S.Reuscher (2017) Adapting genotyping-by-sequencing for rice F2 populations. G3 (Bethesda) 7: 881-893.

Huang, R., L. Jiang, J.Zheng, T. Wang, H. Wang, Y. Huang and Z. Hong (2013) Genetic bases of rice grain shape: so many genes, so little known. Trends Plant Sci. 18: 218-226.

Ishimoto, M. (2007) Effects of the ripening stage and the occurrence of split-hull paddy of rice plant on development of nymphs of the rice leaf bug, Trigonotylus caelestialium (Kirkaldy) (Heteroptera: Miridae). Jpn. J. Appl. Entomol. Zool. 51: 107-114.

Ito, K. (2004) A possible cause of recent outbreaks of rice-ear bugs changes in use of paddy field. Ann. Rept. Plant Prot. North Japan 55: 134-139.

Kavallieratos, N.G., C.G.Athanassiou, F.H.Arthur and J.E. Throne (2012) Lesser grain borers, Rhyzopertha dominica, select rough rice kernels with cracked hulls for reproduction. J. Insect Sci. 12: 38.

Li, N. and Y.Li (2016) Signaling pathways of seed size control in plants. Curr. Opin. Plant Biol. 33: 23-32.

Li, Y., C.Fan, Y.Xing, Y. Jiang, L. Luo, L.Sun, D. Shao, C.Xu, X. Li, J.Xiao et al. (2011) Natural variation in GS5 plays an important role in regulating grain size and yield in rice. Nat. Genet. 43: 1266-1269.

Mao, H., S.Sun, J.Yao, C.Wang, S.Yu, C.Xu, X.Li and Q.Zhang (2010) Linking differential domain functions of the GS3 protein to natural variation of grain size in rice. Proc. Natl. Acad. Sci. USA
107: 19579-19584

McCouch, S.R., L. Teytelman, Y.Xu, K.B. Lobos, K. Clare, M. Walton, B. Fu, R. Maghirang, Z.Li, Y. Xing et al. (2002) Development and mapping of 2240 new SSR markers for rice (Oryza sativa L.). DNA Res. 9: 199-207.

Nagata, K., T.Ando, Y.Nonoue, T.Mizubayashi, N. Kitazawa, A. Shomura, K. Matsubara, N. Ono, R. Mizobuchi, T. Shibaya et al. (2015) Advanced backcross QTL analysis reveals complicated genetic control of rice grain shape in a japonica $\times$ indica cross. Breed. Sci. 65: 308-318.

Shanmugavadivel, P.S., S.V.A. Mithra, C.Prakash, M.K.Ramkumar, R. Tiwari, T.Mohapatra and N.K. Singh (2017) High resolution mapping of QTLs for heat tolerance in rice using a 5K SNP array. Rice 10: 28 .

Shinada,H., T.Yamamoto, E. Yamamoto, K.Hori, J.Yonemaru, S. Matsuba and K. Fujino (2014) Historical changes in population structure during rice breeding programs in the northern limits of rice cultivation. Theor. Appl. Genet. 127: 995-1004.

Singh, N., B. Singh, V. Rai, S. Sidhu, A.K. Singh and N.K. Singh (2017) Evolutionary insights based on SNP haplotypes of red pericarp, grain size and starch synthase genes in wild and cultivated rice. Front. Plant Sci. 8: 972.

Song, X.J., W.Huang, M.Shi, M.Z.Zhu and H.X.Lin (2007) A QTL for rice grain width and weight encodes a previously unknown RING-type E3 ubiquitin ligase. Nat. Genet. 39: 623-630.

Wang, X., Y.Pang, J.Zhang, Z.Wu, K.Chen, J.Ali, G.Ye, J.Xu and Z.Li (2017) Genome-wide and gene-based association mapping for rice eating and cooking characteristics and protein content. Sci. Rep. 7: 17203.

Yamamoto, T., H.Nagasaki, J.Yonemaru, K.Ebana, M.Nakajima, T. Shibaya and M. Yano (2010) Fine definition of the pedigree haplotypes of closely related rice cultivars by means of genome-wide discovery of single-nucleotide polymorphisms. BMC Genomics 11: 267.

Ye, K., M.H. Schulz, Q. Long, R. Apweiler and Z. Ning (2009) Pindel: a pattern growth approach to detect break points of large deletions and medium sized insertions from paired-end short reads. Bioinformatics 25: 2865-2871.

Zaid,I.U., W.Tang, E.Liu, S.U.Khan, H.Wang, E.W.Mawuli and D. Hong (2017) Genome-wide single-nucleotide polymorphisms in CMS and restorer lines discovered by genotyping using sequencing and association with marker-combining ability for 12 yield-related traits in Oryza sativa L. subsp. japonica. Front. Plant Sci. 8: 143.

Zhao, D., P.Li, L.Wang, L. Sun, D. Xia, L.Luo, G. Gao, Q.Zhang and Y.He (2017) Genetic dissection of large grain shape in rice cultivar 'Nanyangzhan' and validation of a grain thickness QTL ( $q$ GT3.1) and a grain length QTL (qGL3.4). Mol. Breed. 37: 42.

Zhen, W., J.Y.Chen, Y.J.Zhu, Y.Y.Fan and N.J.Y.Zhu (2017) Validation of $q G S 10$, a quantitative trait locus for grain size on the long arm of chromosome 10 in rice (Oryza sativa L.). J. Integr. Agric. 16: $16-26$ 